\title{
Mädchen und Jungen - unterschiedliche Fertigkeiten trotz gleicher Fähigkeiten? Ergebnisse aus PISA 2003.
}

\author{
Désirée Burba und Jürgen Rost
}

Gender, Alter, Sozialstruktur und Kriminalitätsbelastung korrelieren eindeutig, und zwar dahingehend, dass Mädchen in allen Bereichen deutlich weniger belastet, bei Körperverletzungen und allen schwereren Delikten geradezu auffällig. Also stellt sich die Frage, ob auch im Bildungsbereich Mädchen Vorteile und manche Jungen deutliche Nachteile aufweisen. Aber dies lässt sich - vom Leseverhalten einmal abgesehen - nicht so eindeutig zeigen.

\section{Einleitung}

Ergebnisse im Rahmen von PISA werden in der Öffentlichkeit oftmals nur als reine Ranginformationen wahrgenommen. So werden Staaten oder Bundesländer hinsichtlich ihrer Leistung verglichen, Schülerleistungen vielleicht noch nach sozioökonomischen Aspekten, Migrationsstatus oder Motivation beurteilt. Geschlechterunterschiede zu eruieren zeigt sich aber als unabdingbar dafür, Fairness und Chancengleichheit über wahrzunehmende Fördermaßnahmen herzustellen. Dabei bietet PISA gerade die Möglichkeit, Geschlechterunterschiede im Vergleich verschiedener Bildungssysteme zu betrachten. Das Augenmerk liegt bei PISA weniger darauf, die Anlage-Umwelt-Debatte zu beantworten, die in der Wissenschaft um Geschlechterunterschiede geführt wird. Die Natur wird wahrscheinlich in allen Ländern gleich wirksam sein. Da PISA eine bildungspolitische Studie ist, geht es mehr darum, zu sehen, wie unterschiedlich die Bildungssysteme in verschiedenen Staaten auf Geschlechterunterschiede wirken. Gerade PISA eignet sich für die Beleuchtung zentraler Geschlechterunterschiede in kognitiven Leistungen, weil viele verschiedene Staaten miteinbezogen wurden und eine hohe Stichprobengröße verlässliche, durch die international abgesicherte Stichprobenziehung repräsentative Aussagen erlaubt.

\section{Geschlechterunterschiede - angeboren, anerzogen oder beides?}

Aus psychologischer Sicht werden Geschlechterunterschiede in kogni- tiven Leistungen aus verschiedenen Perspektiven zu erklären versucht. Halpern (2000) gibt einen Überblick über den biologischen, den psychosozialen und als Kombination aus den beiden den biopsychosozialen Ansatz.

Während biologische Theorien auf den Unterschied aus Genen, Hormonen, Gehirnorganisation und Gehirnentwicklung fokussieren, stellen psychosoziale Ansätze Rollenstereotype oder differenzielle Fähigkeiten in den Vordergrund.

Für jede der verschiedenen Erklärungsansätze gibt es stützende Befunde. So zeigt eine Studie von Hausmann (2000), dass bestimmte kognitive Leistungen von Frauen in Abhängigkeit von Hormonschwankungen ihres Zyklus variieren. Spencer, Steele und Quinn (1999) hingegen konnten zeigen, dass die Mathematik-Leistung von Collegestudentinnen denen ihrer männlichen Kommilitonen entsprach, wenn man bei der Versuchsinstruktion den Teilnehmerinnen und Teilnehmern keine Geschlechterunterschiede vorhersagte, während in der Kontrollbedingung die weiblichen Probanden deutlich schlechter abschnitten.

Bereits die Umwelt-Anlage-Debatte - nicht nur über Unterschiede zwischen Männern und Frauen - in der Psychologie zeigt, dass Biologie und Sozialisation in einander greifen. In diesem Sinne kombbiniert ein psychobiosozialer Ansatz die beiden Richtungen und trägt damit den vielfältigen Befunden auf dem Gebiet der Geschlechterforschung Rechnung (Halpern, 2000). Bei diesem Ansatz wird die Dichotomie zwischen Anlage und Umwelt abgelehnt. Die Effekte werden als un- trennbar und in einander greifend gesehen. Untersuchungen bspw. zu Auswirkungen von Psychotherapie auf das Gehirn (Roffman, Marci, Glick, Dougherty, \& Rauch, 2005) bestätigen eine solche Annahme. Hier konnte gezeigt werden, dass Interaktionen mit der Umwelt sich in der Gehirnorganisation niederschlagen.

Für Schulleistungsstudien steht im Vordergrund, dass Schülerinnen und Schüler differenziert gefördert werden sollten, sofern sich eine Heterogenität feststellen lässt. Je detaillierter die Stärken und Schwächen bekannt sind, desto ressourcenorientierter lässt sich eine Förderung ansetzen. Da Schulleistungen die Grundlage für Berufswahl und die Qualifikation nachfolgender Tätigkeiten bilden, sollten Mädchen wie Jungen die gleichen Chancen haben.

\section{PISA - worum es überhaupt geht}

PISA (Programme for International Student Assessment) ist eine von der OECD (Organisation für wirtschaftliche Zusammenarbeit und Entwicklung) durchgeführte internationale Schulleistungsstudie. Es handelt sich um eine politisch konzipierte Studie, die vergleichende Bildungsindikatoren der Mitglieds- und Partnerstaaten als Grundlage für politische Entscheidungen erfasst.

In den zyklisch angelegten Erhebungswellen (2003, 2003 und 2006) werden die Basiskompetenzen in den Bereichen Lesen, Mathematik und Naturwissenschaften sowie fächerübergreifende Kompetenzen von 15Jährigen in Bildungssystemen erhoben, die als Voraussetzungen nicht nur für den aktuellen und nachfolgenden Bildungsprozess, sondern auch für eine aktive Teilnahme am gesellschaftlichen Leben angenommen werden. In den Erhebungswellen wechselten die Schwerpunkte, so wurde von PISA 2000 das Lesen ausführlicher erfasst, während bei PISA 2003 die Mathematik im Zentrum des Interesses stand, um die Naturwissenschaften bei PISA 2006 umfangreicher zu erheben. PISA ermöglicht als internationale Studie sowohl den Vergleich zwischen Staaten, bietet aber auch Raum für nationale Ergänzungen, von dem in Deutschland hinsichtlich der Messzeitpunkte, Erweiterung der Stichprobe (Ländervergleich innerhalb Deutschlands sowie Testung ganzer Klassen) sowie des Einsatzes eines national entwickelten Instrumentariums Gebrauch gemacht wurde. Einen detaillierten Überblick über Aufbau und Ergebnisse der Studie geben die internationalen Veröffentlichungen (OECD, 2003; OECD, 2004; OECD, 2005). Für die Darstellung der PISA-Studie aus deutscher Perspektive sei hier auf die nationalen Publikationen (Baumert et al., 2001; Prenzel et al., 2004) verwiesen.

\section{Sind Jungen die Verlierer bei PISA?}

Sofern nicht anders angegeben, beziehen sich die hier referierten Geschlechterunterschiede vor allem auf PISA 2003. Zunächst soll auf die Leistungsaspekte eingegangen werden, um dann einen kurzen Überblick über die motivationalen und emotionalen Aspekte zu geben.

Insgesamt lässt sich sagen, dass es den verschiedenen PISA-Teilnehmerstaaten in unterschiedlicher Weise 
gelingt, Geschlechterunterschiede zu minimieren. Dabei muss zum einen berücksichtigt werden, wie bedeutend die Geschlechterunterschiede sind bzw. wie stark diese ausgeprägt sind, aber auch auf welchem Niveau sich die Leistung der Schülerinnen und Schüler bewegt. Bei PISA 2003 sind im Durchschnitt über alle OECD-Staaten hinweg in der Leseleistung etwa mittelgroße Unterschiede zugunsten der Mädchen zu verzeichnen, die Unterschiede in Mathematik und Naturwissenschaften sind als gering zugunsten der Jungen $\mathrm{zu}$ beurteilen. Im fächerübergreifenden Problemlösen sind keine bedeutsamen Geschlechterunterschiede zu verzeichnen.

Auf der Ebene der einzelnen Staaten zeigen sich unterschiedlichste Muster. Ein eindeutiges Bild liefert die Lesekompetenz, hier sind durchweg mittlere bis hohe Geschlechterunterschiede zu verzeichnen, die alle zugunsten der Mädchen ausfallen, in jedem Staat schneiden also Mädchen im Lesen besser ab als Jungen. Es zählen auch wesentlich mehr Jungen zur Risikogruppe im Lesen, also zur Gruppe der kompetenzschwachen Leser. Im Durchschnitt sind hier also die Jungen im Nachteil, denn die Lesekompetenz kann als Voraussetzung für Lernleistungen in anderen Domänen angenommen werden.

Im Bereich der Mathematik zeichnet sich ein anderes Bild ab: Hier sind in immerhin sieben der 31 OECDStaaten keine Geschlechterunterschiede zu beobachten und in Island schneiden sogar die Mädchen besser $\mathrm{ab}$ als die Jungen. In den restlichen Staaten (darunter auch Deutschland) zeigen die Jungen bessere Leistungen, wenn es sich auch nicht um so deutliche Geschlechterunterschiede wie im Bereich Lesen handelt, man kann nur von geringen Unterschieden sprechen.

Im Bereich der Naturwissenschaften ist eine pauschale Aussage noch weniger möglich. In zwei der 31 OECD-Staaten zeigen die Mädchen statistisch bedeutsam bessere Leistungen, und zwar in Island und Finnland. In elf Staaten hingegen weisen die Jungen eine höher naturwissenschaftliche Kompetenz auf. Bei den restlichen 16 Staaten (wie auch Deutschland) gibt es keine Geschlechterunterschiede. Die auftre- tenden Effekte hinsichtlich der naturwissenschaftlichen Kompetenz sind als gering $\mathrm{zu}$ beurteilen. Die Leistungen im Problemlösen geben ein homogenes und so geschlechterfaires Bild ab. Hier sind in fast allen Staaten keine Unterschiede zwischen den Leistungen von Jungen und Mädchen auszumachen. Lediglich in Island, Schweden und Norwegen ist die Problemlösekompetenz der Mädchen höher ausgeprägt als die der Jungen.

Wie bei Leutner, Klieme, Meyer, \& Wirth (2004) ausgeführt, zeigt die Problemlösekompetenz das kognitive Potential eines Schülers an, das für die komplexen Problemlöseprozesse außerhalb von Unterricht und Schule aber auch für die mathematische Kompetenz im PISA-Test benötigt wird. Eine Differenz zwischen den Kompetenzbereichen Problemlösen und Mathematik bedeutet demnach, dass das allgemeine kognitive Potenzial aus verschiedenen Gründen nicht ausgeschöpft werden kann.

Da in den meisten Staaten keine Geschlechterunterschiede im Problemlösen, zugleich aber in vielen Staaten statistisch bedeutsame Unterschiede in der Mathematikkompetenz zugunsten der Jungen zu verzeichnen sind, spricht das dafür, dass in diesen Ländern das allgemeine kognitive Potenzial der Mädchen nicht hinreichend ausgeschöpft ist, das heißt, dass sie bessere Leistungen in Mathematik erzielen könnten (siehe dazu Zimmer, Burba, \& Rost (2004)). Deutschland gehört zu diesen Ländern.

Bezüglich des Leistungsniveaus zeigte sich bei PISA 2000 (Stanat \& Kunter, 2001), dass hohe Gesamtleistungen mit hohen Geschlechterunterschieden einhergehen. Während bei PISA 2003 dieses Bild Finnland und Korea stützen, lassen sich immerhin drei Staaten ausmachen, die insgesamt überdurchschnittliche Leistungen in allen Kompetenzbereichen aufweisen und in denen Jungen und Mädchen in Mathematik, Naturwissenschaften und Problemlösen gleiche Leistungen erbringen. Dazu zählen die Niederlande, Belgien und Australien. Die Unterschiede im Lesen zugunsten der Mädchen sind jedoch auch in diesen Ländern mehr oder weniger stark ausgeprägt. Japan reiht sich ebenfalls in diese Befundlage mit ein, allerdings sind hier die Leseleistungen insgesamt nur auf durchschnittlichem Niveau.

Es zeigt sich also, dass es durchaus gelingen kann und einigen Ländern auch gelungen ist, die Unterschiede zwischen den Geschlechtern auszugleichen und gleichzeitig ein hohes Leistungsniveau zu erreichen. Beim Lesen hingegen muss eine Minimierung der geschlechtlichen Heterogenität erst noch erreicht werden.

Neben den kognitiven Leistungen wurden bei PISA auch motivationale und emotionale Aspekte erfasst. In Deutschland zeigt sich bei PISA 2003, dass hinsichtlich der Selbsteinschätzungen der Schülerinnen und Schüler in bezug auf Mathematik noch immer Geschlechterunterschiede bestehen. Dies werden zwar geringer, wenn die Kompetenz steigt, jedoch sind die Unterschiede auch in der Gruppe der Leistungsstarken noch immer ausgeprägt (Zimmer et al., 2004). Bemerkenswert ist, dass also bei vergleichbaren Leistungen die Selbsteinschätzungen der Jungen und Mädchen deutlich in stereotyper Weise auseinanderklaffen.

Dass Jungen stärker an Mathematik interessiert sind, findet sich bei einer großen Anzahl von Staaten (OECD, 2004), Tab 3.1). Bei PISA 2000 (Stanat \& Kunter, 2001) zeigte sich zudem, dass die Einstellung der Jungen zum Lesen deutlich negativer ist als bei Mädchen, was sich allerdings im Gegensatz $\mathrm{zu}$ den Ergebnissen in Mathematik bei PISA 2003 deutlicher in den Leistungen widerspiegelte. Dies ist insofern von Interesse, als dass Emotion und Motivation meist eng mit der Leistung gekoppelt sind (Pekrun et al., 2004; Pekrun \& Zirngibl, 2004) und sich auch auf Kursund Berufswahl auswirken. Da Mädchen und Frauen noch immer in vielen Berufen unterrepräsentiert sind und Jungen in der Schlüsselkompetenz Lesen bedeutsam hinter den Leistungen der Mädchen zurückfallen, ist es eine dringliche Aufgabe der Schule und Gesellschaft, Chancengleichheit zu fördern, indem sowohl Leistungs- als auch motivationale und emotionale Aspekte berücksichtigt werden.

Zusammenfassend lässt sich sagen, dass noch immer Geschlechterun- terschiede sowohl hinsichtlich der Leistung als auch emotionaler und motivationaler Aspekte unter den 15-jährigen in Bildungssystemen zu verzeichnen sind. Diese Geschlechterunterschiede sind als klein bis mittelgroß zu beurteilen und erreichen nur vereinzelt größere Ausmaße. Über den Kompetenzbereich Lesen lässt sich eine einheitliche Aussage treffen, hier sind die Unterschiede am größten und stets zugunsten der Mädchen, während in Mathematik und den Naturwissenschaften die Ergebnisse klein und uneinheitlich sind. Im Problemlösen, dem Indikator für das allgemeine kognitive Potenzial, gibt es fast durchweg keine Unterschiede zwischen Mädchen und Jungen. Einige Länder schaffen es besser als andere, die Unterschiede zwischen Jungen und Mädchen auf einem gleichzeitig hohen Gesamtniveau zu reduzieren oder sogar ganz zu beseitigen, allerdings bleiben die Unterschiede im Lesen in allen Ländern statistisch bedeutsam. Während es also ein deutliches Jungenproblem in der Lesekompetenz auszugleichen gilt, sollten die Mädchen hinsichtlich ihres Interesses und ihrer Emotionen gegenüber der Mathematik gefördert werden, da sie nachweislich das kognitive Potential für höhere Leistungen, auch für Mathematik, aufweisen.

Désirée Burba, Jürgen Rost, IPN an der Universität Kiel.

\section{Literatur}

Baumert, J., Klieme, E., Neubrand, M., Prenzel, M., Schiefele, U., Schneider, W., Stanat, P., Tillmann, K.-J., \& Weiß, M. (Hrsg.). (2001). PISA 2000. Basiskompetenzen von Schülerinnen und Schülern im internationalen Vergleich. Opladen: Leske + Budrich.

Halpern, D. (2000). Sex differences in cognitive abilities (3rd edition. Aufl.). Mahwah, NJ: Erlbaum.

Hausmann, M. (2000). Steroidmodulation funktioneller zerebraler Asymmetrien. Bochum: Ruhr-Universität.

Leutner, D., Klieme, E., Meyer, K., \& Wirth J. (2004). Problemlösen. In M. Prenzel, J. Baumert, W. Blum, R. Lehmann, D. Leutner, M. Neubrand, R. Pekrun, H.G. Rolff, J. Rost, \& U. Schiefele (Hrsg.), PISA 2003. Der Bildungsstand der Jugendlichen in Deutschland - Ergebnisse des zweiten internationalen Vergleichs (S. 147 175). Münster: Waxmann.

OECD. (2003). The PISA 2003 Assessment Framework-Mathematics, reading, scien- 
ce and problem solving knowledge and skills. Paris: OECD.

OECD. (2004). Lernen für die Welt von morgen. Erste Ergebnisse von PISA 2003. Paris: OECD.

OECD. (2005). PISA 2003 Technical Report. Paris: OECD.

Pekrun, R., Götz, T., Vom Hofe, R., Blum, W., Jullien, S., Zirngibl, A., Kleine, M., Wartha, S., \& Jordan, A. (2004). Emotionen und Leistung im Fach Mathematik: Ziele und erste Befunde aus dem »Projekt zur Analyse der Leistungsentwicklung in Mathematik« (PALMA). In J. Doll \& M. Prenzel (Hrsg.), Bildungsqualität von Schule. Lehrerprofessionalisierung, Unterrichtsentwicklung und Schü- lerförderung als Strategien der Qualitätsverbesserung. Münster: Waxmann.

Pekrun, R., \& Zirngibl, A. (2004). Schülermerkmale im Fach Mathematik. In M. Prenzel, J. Baumert, W. Blum, R. Lehmann, D. Leutner, M. Neubrand, R. Pekrun, H.-G. Rolff, J. Rost, \& U. Schiefele (Hrsg.), PISA 2003. Der Bildungsstand der Jugendlichen in Deutschland-Ergebnisse des zweiten internationalen Vergleichs (S. 191-210). Münster: Waxmann.

Prenzel, M., Baumert, J., Blum, W., Lehmann, R., Leutner, D., Neubrand, M., Pekrun, R., Rolff, H.-G., Rost, J., \& Schiefele, U. (2004). PISA 2003. Der Bildungsstand der Jugendlichen in Deutsch-
land-Ergebnisse des zweiten internationalen Vergleichs. Münster: Waxmann.

Roffman, J. L., Marci, C. D., Glick, D. M., Dougherty, D. D., \& Rauch, S. L. (2005). Neuroimaging and the functional neuroanatomy of psychotherapy. Psychological Medicine, 35(10), 1385 1398.

Spencer, S. J., Steele, C. M. \& Quinn, D. M. (1999). Stereotype threat and women's math performance. Journal of Experimental Social Psychology, 35, 4-28.

Stanat, P., \& Kunter, M. (2001). Geschlechterunterschiede in Basiskompetenzen. In J. Baumert, E. Klieme, M. Neubrand, M. Prenzel, U. Schiefele, W. Schneider, P. Stanat, K.-J. Tillmann, \&
M. Weiß (Hrsg.), PISA 2000. Basiskompetenzen von Schülerinnen und Schülern im internationalen Vergleich (S. 249 269). Opladen: Leske + Budrich.

Zimmer, K., Burba, D., \& Rost, J. (2004). Kompetenzen von Jungen und Mädchen. In M. Prenzel, J. Baumert, W. Blum, R. Lehmann, D. Leutner, M. Neubrand, R. Pekrun, H.-G. Rolff, J. Rost, \& U. Schiefele (Hrsg.), PISA 2003. Der Bildungsstand der Jugendlichen in Deutschland-Ergebnisse des zweiten internationalen Vergleichs (S. 211-223). Münster: Waxmann.

\section{Frank Winter über Verena Sabaß zu Schülergremien}

Verena Sabaß legte ihre Arbeit, die 2003 abgeschlossen wurde, in der Reihe Kriminalpolitische Schriften, Band 2 beim LIT Verlag vor. Bei ihrer Arbeit handelt es sich um eine Dissertation der Juristischen Fakultät der Ludwig-Maximillians-Universität München. Dieser Hinweis soll übermäßige Erwartungen an eine soziologisch-psychologische Ausrichtung der Veröffentlichung begrenzen.

Im November 2000 entstand auf Initiative des Bayrischen Justizministeriums mit dem »Kriminalpädagogischen Schülerprojekt « (KPS) im Amtsgerichtsbezirk Aschaffenburg das in Deutschland damals neuartige und reine Diversionsmodell für junge Straftäter, dessen Kern es ist, die ausgewiesenermaßen positiven Einflüsse jugendlicher Peers auf ihre straffällig gewordenen Altersgenossen für die Jugendstrafrechtspflege nutzbar zu machen. In geeigneten Fällen »leichter bis mittlerer Jugendkriminalität « (Seite 3), die ansonsten im Erziehungs- oder vereinfachten Jugendverfahren erledigt würden, wird Beschuldigten nach staatsanwaltschaft- licher Anregung die Möglichkeit gewährt, »freiwillig « mit einem »Gremium aus drei dafür ausgebildeten Schülern« Gespräche über die begangene Straftat zu führen (3). Im Anschluss und als Essenz dieser Gespräche vereinbaren die Jugendlichen »eine weitere Leistung «, die der Beschuldigte erbringen soll, »um die Einsicht in das Unrecht der Tat zu erhöhen « bzw. es » wieder gut zu machen «(3). Gelingt eine Einigung und wird die Leistung wie vereinbart erbracht, »sieht die Staatsanwaltschaft regelmäßig nach § 45 Abs. 2 JGG von der weiteren Verfolgung $a b \ll$ (3).

Die Bayrische Initiative ist von den amerikanischen teen-courts inspiriert und wurde von Beginn an wissenschaftlich begleitet. Sabaß beginnt ihren Band entsprechend mit einer Einführung zur sozialisatorischen Bedeutung Gleichaltriger in der Jugendphase (5 ff) und legt anschließend Funktionsweise, Verbreitung und Forschungsstand zu den teen-courts dar (27 ff), wobei sie auch die Peermediation an Schulen streift (19 f). Die Frage der Übertragbarkeit des amerikanischen Modells (66 ff) wird mit den rechtlichen Rahmenbedingungen des KPS gekoppelt, ehe die empirischen Untersuchungen zu Art und Zahl der im KPS bearbeiteten Fälle, zur Akzeptanz des KPS bei den Beschuldigten, zu Ausbildung und Motiven der ausgebildeten Gremiumsschüler sowie zu den Gesprächen und den darin ausgehandelten Maßnahmevereinbarungen (152 ff) mit den Beschuldigten folgen. Eine Rückfallstudie (180ff) sowie Interviews (169 ff) mit Beschuldigten und Gremiumsmitgliedern runden den Band ab, der einer Stellungnahme zur Zukunftsfähigkeit dieser Diversionsmaßnahme schließt (195) und durch einen umfänglichen Anhang ergänzt wird, der u. a. auch Konzeption und Form- und Infoblätter des Aschaffenburger Modells enthält und damit den Aufbau anderer Projekte erleichtert.

Bundesarbeitsgemeinschaft für Straffälligenhilfe e. V. hat ihre Geschäftsstelle in Bonn wieder in betrieb genommen.

Nähere Info: info@straffaelligenhilfe.de

Am 28. 09. 2006 findet eine Fachkonferenz in der Justizvollzugsanstalt in 94315 Straubingen, Grasiger Weg 44 statt.

Thema:

Soziale Arbeit im Strafvollzug des 21. Jahrhundert.

kontakt@bag-sozialarbeit.dea 